\title{
Non-rigid Registration of 3D Ultrasound Images of Brain Tumours Acquired during Neurosurgery
}

\author{
Marloes M.J. Letteboer ${ }^{1}$, Peter W.A. Willems ${ }^{2}$, Max A. Viergever ${ }^{1}$, and \\ Wiro J. Niessen ${ }^{1}$ \\ ${ }^{1}$ Image Sciences Institute, University Medical Center, Utrecht, The Netherlands \\ \{marloes, max, wiro\}@isi.uu.nl; \\ ${ }^{2}$ Department of Neurosurgery, University Medical Center, Utrecht, \\ The Netherlands P.Willemsaneuro.azu.nl
}

\begin{abstract}
Intraoperative brain deformation is one of the most contributing factors to the inaccuracy of image-guided neurosurgery systems. One option for correcting for this deformation is to acquire 3D ultrasound images during surgery and use these to update the information provided by the preoperatively acquired MR. To compare ultrasound volumes at different stages of surgery, non-rigid registration techniques are necessary. We present the results of applying a non-rigid registration algorithm, based on free-form deformations using B-splines and using normalized mutual information as a similarity measure, to 3D ultrasound volumes of two patients with brain tumours. For these two patients we registered an ultrasound volume acquired prior to opening the dura with an ultrasound volume acquired after opening the dura, but prior to surgery. When comparing the segmented tumours after affine registration plus free-form registration with a control point spacing of $4 \mathrm{~mm}$ to the segmented tumour volumes after registration with the image-guided surgery system, the volume overlap increased from approximately $76 \%$ to $96 \%$ for both patients.
\end{abstract}

\section{Introduction}

In image-guided neurosurgical interventions the position of the tumour is determined by navigation based on preoperatively acquired MR data. In the image-guided surgery systems that are currently available it is assumed that no brain deformation occurs during the intervention. However, brain deformations of up to $10 \mathrm{~mm}$ have been reported [1,2]. As a consequence, the tumour location and shape with respect to the preoperative MR is uncertain. To correct for these deformations of the tumour and surrounding brain tissue, intraoperatively acquired ultrasound data can be used $[3,4]$ in combination with the preoperatively acquired MR data.

We use a commercially available navigation system to track the ultrasound probe while acquiring 2D ultrasound data during neurosurgery. Since we now have a series of $2 \mathrm{D}$ ultrasound images with their corresponding positions we can reconstruct a 3D ultrasound volume [5]. The first ultrasound volume is acquired just after craniotomy, prior to opening the dura. It is assumed that little or no brain deformation has occurred between acquiring the preoperative MR and this first ultrasound volume. 
Therefore, the task of registering the preoperative MR with intraoperative ultrasound volumes can be replaced by the easier task of registering the ultrasound volume acquired prior to opening the dura with the subsequent ultrasound volumes. In order to detect brain deformations occurring in the course of surgery, especially after opening the dura, ultrasound volumes acquired at different stages of surgery have to be compared. To calculate the deformation between subsequent ultrasound volumes non-rigid registration techniques are necessary.

In literature several methods for non-rigid registration have been reported [6,7]. However, the majority of these methods have not been developed for ultrasound registration tasks. Although the use of these non-rigid registration methods on ultrasound volumes is not very common, some reports are available on this subject $[8,9]$.

In this paper we apply a non-rigid registration algorithm, based on free-form deformations (FFD's) using B-splines and using normalized mutual information as a similarity measure, to 3D ultrasound volumes of brain tumours.

\section{Materials and Methods}

\subsection{Data Acquisition}

During image-guided neurosurgery the ultrasound probe was tracked, using a Polaris camera, and the relative positions of the 2D scans measured were used to reconstruct a 3D volume, using the software package StackSX [10]. For two patients at least two ultrasound volumes were acquired; one prior to opening the dura and one after opening the dura, but prior to tumour removal. It is expected that the brain will deform between these two scans and non-rigid registration techniques will be needed to match them.

For the first patient, $120 \mathrm{~B}$-scans were acquired for both datasets from which a 3D volume was reconstructed. The scans were acquired with a probe penetration depth of $6 \mathrm{~cm}$. The reconstructed volumes used for registration had a matrix size of 150 by 150 by 100 voxels, with a voxels size of 0.5 by 0.5 by $0.5 \mathrm{~mm}$.

For the second patient, 120 B-scans acquired made for both datasets. The scans were acquired with a probe penetration depth of $10 \mathrm{~cm}$. The reconstructed volumes used for registration had a matrix size of 125 by 125 by 100 voxels with a voxel size of 1.0 by 1.0 by $1.0 \mathrm{~mm}$.

\subsection{Non-rigid Registration}

\section{Registration Algorithm}

The goal of our registration process is to find the optimal transformation $T:(x, y, z) \mapsto\left(x^{\prime}, y^{\prime}, z^{\prime}\right)$, which maps all points of the ultrasound image $I(x, y, z, t)$, acquired at time $t$ in the course of surgery, to the ultrasound image $I\left(x^{\prime}, y^{\prime}, z^{\prime}, t_{0}\right)$, taken prior to opening the dura. In general, the deformation of the brain between these two acquisitions is non-rigid, which means affine transformations alone are not sufficient to correct for this motion. Therefore we used the program introduced by Rueckert et al.[6] for the application of breast MR images. 
The algorithm consists of a combined global and local motion model at each image point $(x, y, z)$, and can be expressed as

$$
T(x, y, z)=T_{\text {global }}(x, y, z)+T_{\text {local }}(x, y, z)
$$

The global motion model describes the overall motion of the brain using an affine transformation. An additional local transformation is required to model local deformations in the brain. This local transformation is based on a free-form deformation (FFD), based on B-splines. The basic idea of FFD's is to deform an object by manipulating the underlying mesh of control points. The resulting deformation controls the shape of the 3D object and produces a smooth transformation.

The control points act as parameters of the B-spline FFD and the degree of nonrigid deformation that can be modelled depends on the resolution of the mesh of control points. A large spacing of control points allows modelling of global non-rigid deformations, while a small spacing of control points allows modelling of more local non-rigid deformations. In order to achieve the best compromise between the degree of non-rigid deformation required to model the brain deformation and the associated computational cost a hierarchical multi-resolution approach is used in which the resolution of the control mesh is increased, along with the image resolution, in a coarse to fine fashion.

\section{Parameter Settings}

We registered 3D ultrasound volumes acquired prior to opening the dura with $3 \mathrm{D}$ ultrasound volumes acquired after opening the dura, but prior to surgery, for two patients. The program used for registration has the possibility of choosing several parameters for registration.

A similarity criterion has to be defined, which measures the degree of alignment between the two images. We choose normalized mutual information as the similarity measure, with a histogram bin size of 128 because this measure performed best in our earlier study on rigid 3D ultrasound registration [11].

For each dataset we performed an affine transformation, and affine transformations plus a free-form deformation with a control point spacing of respectively $32,16,8$ and $4 \mathrm{~mm}$.

\subsection{Registration Validation}

To assess the quality of the registration algorithm we calculated the correlation coefficient

$$
C C=\frac{\sum\left(I\left(t_{0}\right)-\bar{I}\left(t_{0}\right)\right) \cdot(T(I(t))-T(\bar{I}(t)))}{\sqrt{\sum\left(I\left(t_{0}\right)-\bar{I}\left(t_{0}\right)\right)^{2} \cdot \sum(T(I(t))-T(\bar{I}(t)))^{2}}}
$$

Here $\bar{I}\left(t_{0}\right), \bar{I}(t)$ denote the average intensities of the images before and after opening the dura and the summation included all voxels within the overlap of both images. 

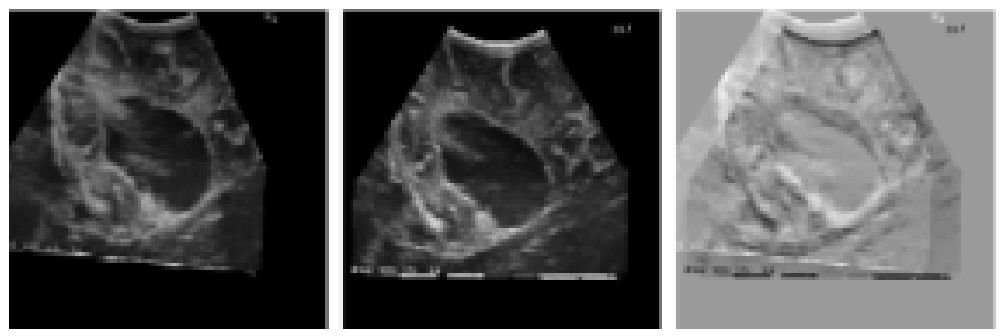

Fig. 1. Data for patient 1. Left: 2D plane of the 3D ultrasound volume acquired prior to opening the dura. Middle: Corresponding 2D plane of the 3D volume acquired after opening the dura. Right: Difference image without additional registration
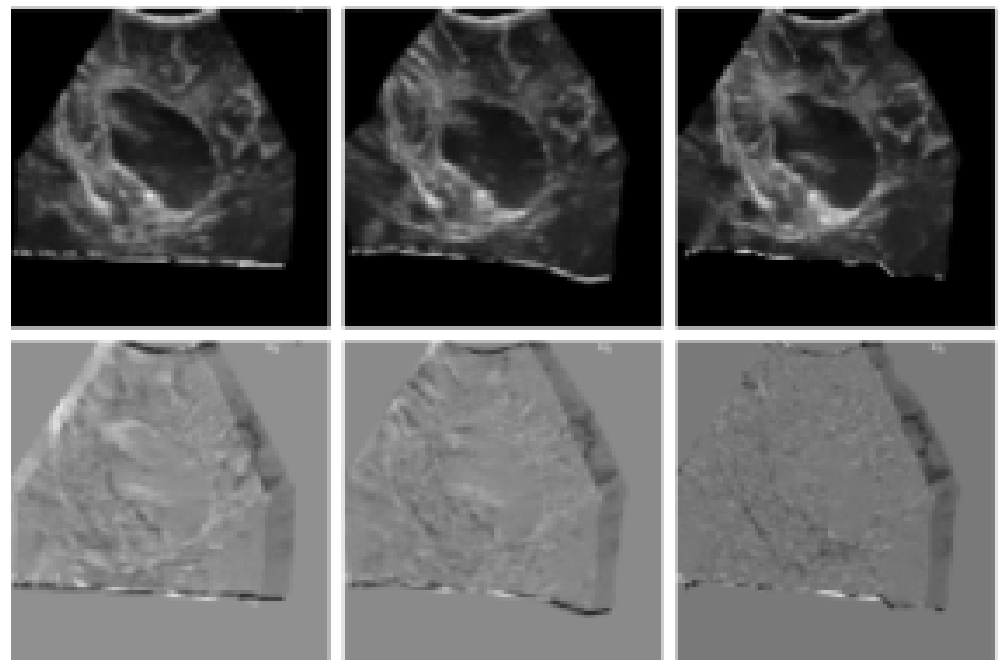

Fig. 2. Registration of the ultrasound volume acquired after opening the dura to the ultrasound volume acquired prior to opening the dura for patient 1. Upper images: Registered volumes, Lower images: Difference images. Left: Affine registration. Middle: Affine registration + FFD with a control point spacing of $16 \mathrm{~mm}$. Right: Affine registration + FFD with a control point spacing of $4 \mathrm{~mm}$

Another method we used to compare the registered image with the initial image is to segment the tumour in both images and calculate the overlap. The overlap is defined as

$$
\text { Overlap }=\frac{V_{1} \cap V_{2}}{\left(V_{1}+V_{2}\right) / 2}
$$

The tumours were segmented using the interactive multi-scale watershed segmentation program Nabla Vision [12], which we used earlier to segment brain tumours in MR images [13].

Since the acquired volumes will not provide information over the exact same brain area, and since the only available information is in the ultrasound beam, all validation will be done on data that is available in both volumes. 

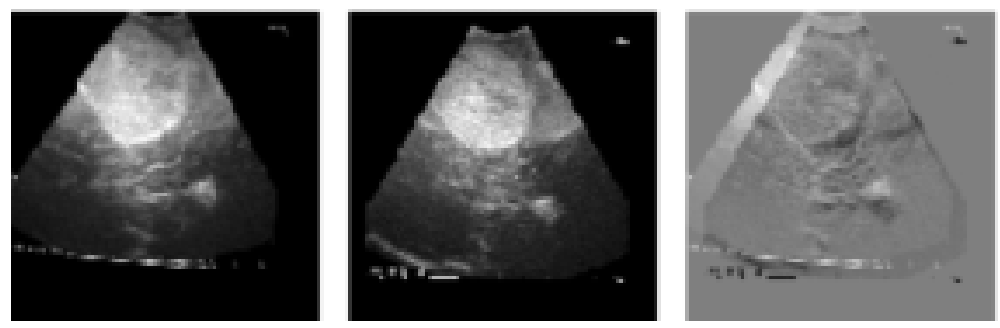

Fig. 3. Data for patient 2. Left: 2D plane of the 3D ultrasound volume acquired prior to opening the dura. Middle: Corresponding 2D plane of the 3D volume acquired after opening the dura. Right: Difference image without additional registration
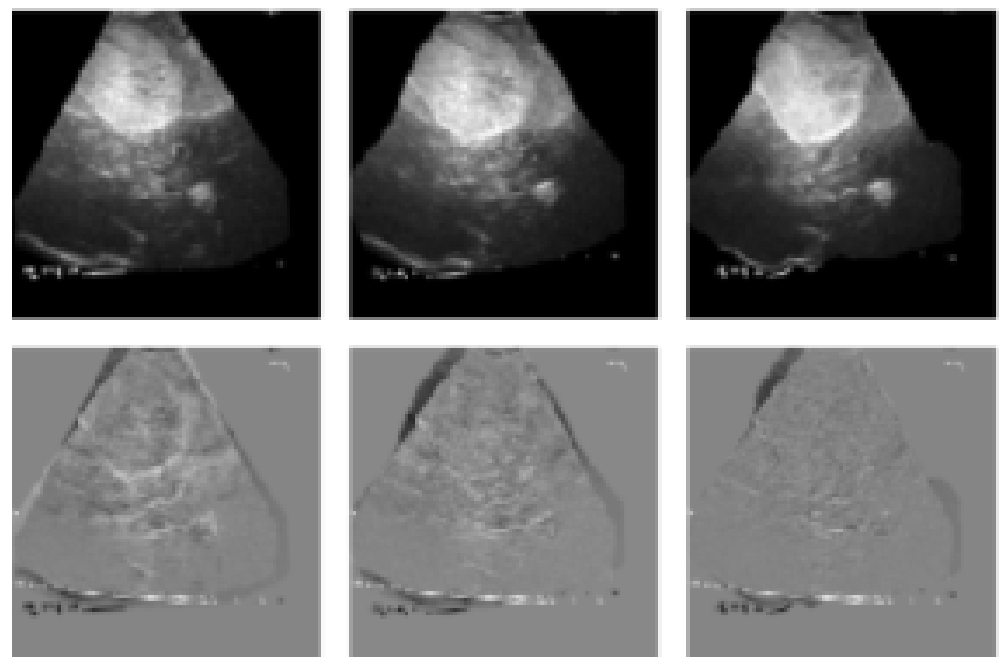

Fig. 4. Registration of the ultrasound volume acquired after opening the dura to the ultrasound volume acquired prior to opening the dura for patient 2. Upper images: Registered volumes, Lower images: Difference images. Left: Affine registration. Middle: Affine registration + FFD with a control point spacing of $16 \mathrm{~mm}$. Right: Affine registration + FFD with a control point spacing of $4 \mathrm{~mm}$

\section{Results and Discussion}

The non-rigid registration algorithm described was applied to 3D ultrasound volumes acquired before and after opening the dura of two patients with brain tumours. These two datasets were selected because of the good visibility of the tumours. For both datasets the results prior to registration, after affine registration (with nine degrees of freedom) and after affine plus free-form deformation with a control point spacing of 32, 16, 8 and $4 \mathrm{~mm}$ were examined.

Figure 1 and 3 show the volumes acquired prior to opening the dura and after opening the dura, but prior to surgery. The initial registration between these images is 
based on the transformation provided by the image-guided neurosurgery system (IGS). In the difference image it can be seen that a deformation of the brain has occurred.
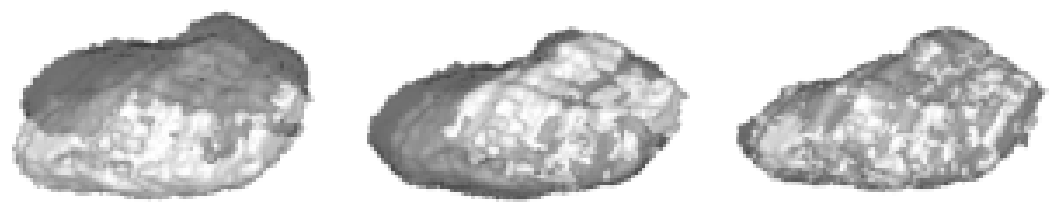

Fig. 5. Overlap between the segmented tumour volume before opening the dura (light grey) to the tumour volume after opening the dura (dark grey). Left: Registration of image-guided surgery system. Middle: Affine registration. Right: Affine + FFD registration with a control point spacing of $4 \mathrm{~mm}$

Table 1. Correlation coefficient and segmented volume overlap, calculated between the ultrasound volume acquired prior to opening the dura to the volume acquired after opening the dura, after registration

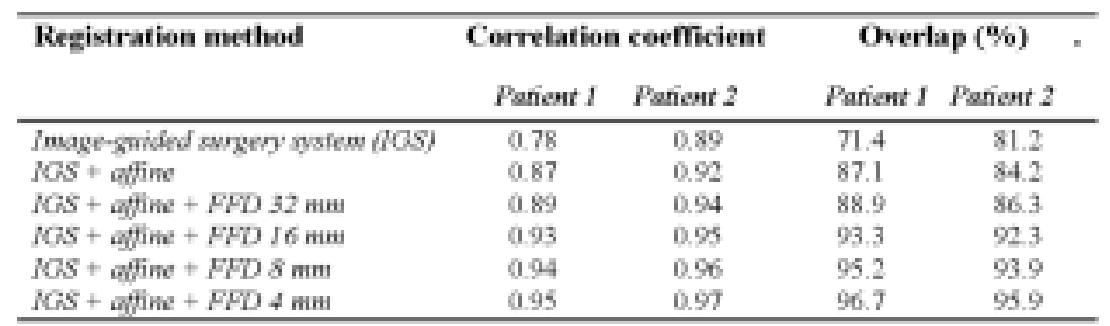

Figure 2 and 4 show the results of the registration process for both patients. The results after affine registration, after affine plus FFD with a control point spacing of $16 \mathrm{~mm}$ and, respectively, $4 \mathrm{~mm}$ are shown. From visual inspection of Figure 2 and 4 it can be concluded that the correspondence between the initial volume and the registered volume does improve when using FFD's with small control point spacing. To quantify this improvement the correlation ratio between the initial image and the registered images is calculated. From Table 1 it can be seen that the correlation between the volumes improves with smaller control point spacing. Because of computational considerations the smallest control point spacing we used was $4 \mathrm{~mm}$. The results may improve when going to even smaller control point spacings.

To validate the registration for our region of interest, the tumour position, a segmentation of the tumour is performed (Figure 5) from which the overlap between the volumes is calculated (Table 1). For the smallest control point spacing used the overlap is best. For patient 1 the overlap improves from $71.4 \%$, with IGS registration, to $87.1 \%$, for affine registration, to $96.7 \%$, for affine registration plus FFD with a control point spacing of $4 \mathrm{~mm}$. For patient 2 the overlap improves from $81.2 \%$, with IGS registration, to $84.2 \%$, for affine registration, to $95.9 \%$, for affine registration plus FFD with a control point spacing of $4 \mathrm{~mm}$. The $4 \%$ of the volume that does not 
overlap after registration are not only due registration errors but also segmentation errors.

Besides the qualitative validation with the correlation coefficient and volume overlap, it is also important that the deformation fields are plausible. By visual inspection of Figure 6, where the affine deformation field and the deformation field for the FFD with a control point spacing of $4 \mathrm{~mm}$ for patient 1 are given, the deformation fields seem to be consisted with the deformations we see between these two images.

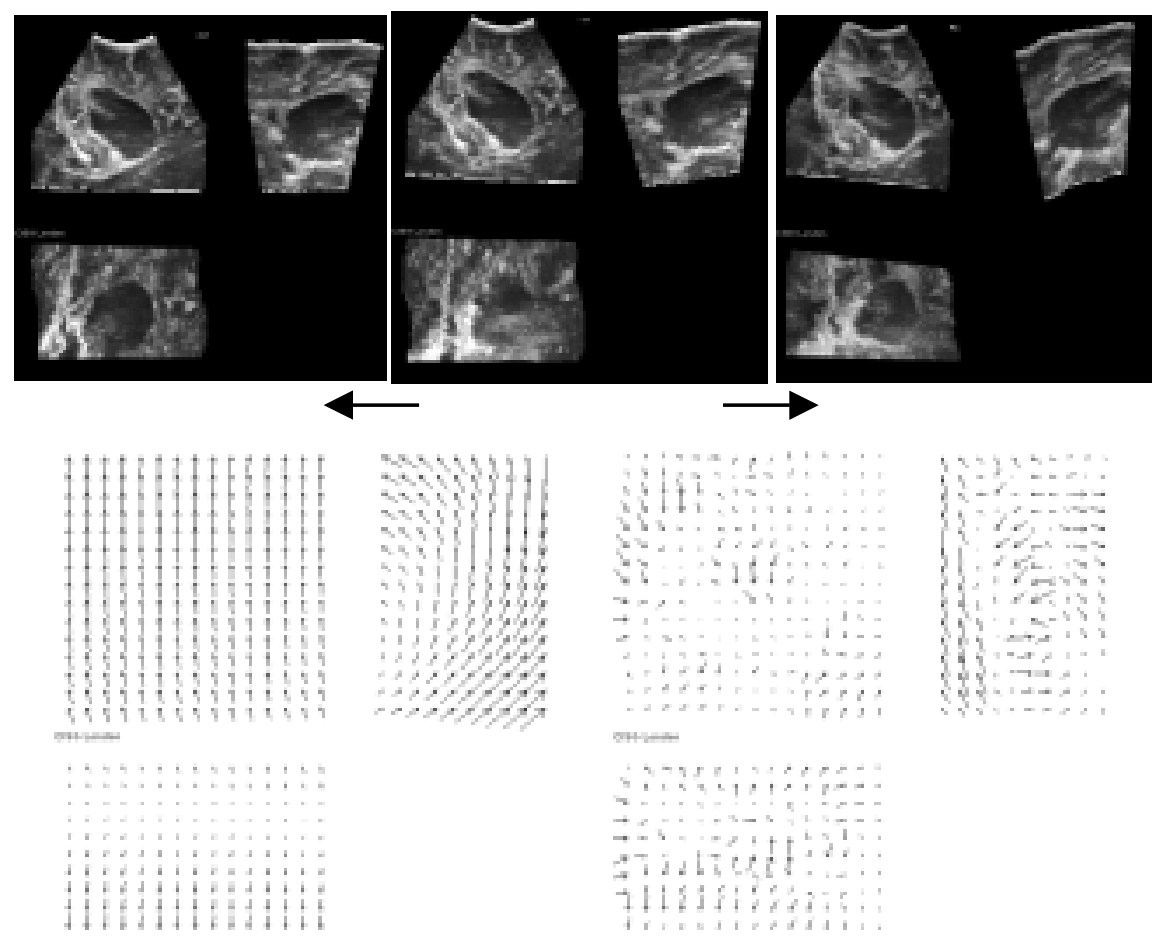

Fig. 6. Deformation field. Top: left: Ultrasound volume after opening the dura, middle: Ultrasound volume after opening the dura + affine registration, right: Ultrasound volume before opening the dura. Bottom: left: Deformation field for affine registration, right: Deformation field for free-form deformation (control point spacing $4 \mathrm{~mm}$ ) without the affine component

\section{Conclusion}

We applied a non-rigid registration algorithm based on free-form deformations using B-splines and using normalized mutual information as a similarity measure, to 3D ultrasound volumes of two patients with brain tumors. We acquired at least two ultrasound datasets for these patients during an image-guided neurosurgical intervention, one prior to opening the dura and one after opening the dura, but prior to surgery. 
The registration was validated by calculating the correlation coefficient between the registered images and by calculating the volume overlap of the tumors. Free-form deformation with a small control point spacing improved the volume overlap of the tumor from an average of $76 \%$ after the registration with the image-guided surgery system to $96 \%$ after registration with FFD. This shows the potential for intraoperative 3D ultrasound acquisition in combination with preoperative MR acquisition for improving the accuracy in image-guided surgery.

\section{References}

1. C.R. Maurer, D.G.L. Hill, C.L. Truwit. Investigation of Intraoperative Brain Deformation Using a 1.5 T Interventional MR System: Preliminary Results. In: IEEE Transactions on Medical Imaging, Vol. 17, No. 5, pp. 817-826, 1998.

2. D.W. Roberts, A. Hartov, F.E. Kennedy, M.I. Miga, K.D. Paulsen. Intraoperative Brain Shift and Deformation: a Quantitative Analysis of Cortical Displacement in 28 Cases. In: Neurosurgery, Vol. 43, No. 4, pp. 749-758, 1998.

3. R.D. Buchholz, D.D. Yeh, P. Kessman. The Correction of Stereotactic Inaccuracy Caused by Brain Shift Using an Intraoperative Ultrasound Device. In: MICCAI Proceedings Lecture notes in Computer Science 1496, Editors: W.M. Wells, A. Colchester, S. Delp, pp. 459-466, Publisher: Springer-Verlag, Cambridge, 1998.

4. R.M. Comeau, A.F. Sadikot, A. Fenster, T.M. Peters. Intraoperative Ultrasound for Guidance and Tissue Shift Correction in Image-Guided Neurosurgery. In: Medical Physics, Vol. 27, No. 4, pp. 787-800, 2000.

5. M.M.J. Letteboer, P.W.A. Willems, P. Hellier, W.J. Niessen. Acquisition of 3D Ultrasound Images During Neuronavigation. In: Computer Assisted Radiology and Surgery, Editors: H.U. Lemke, W.M. Vannier, K. Inamura, A.G. Farman, K. Doi, J.H.C. Reiber, pp. 121-126, Publisher: Springer, Paris, 2002.

6. D. Rueckert, L.I. Sonoda, C. Hayes, D.L. Hill, M.O. Leach, D.J. Hawkes. Nonrigid Registration Using Free-form Deformations: Application to Breast MR Images. In: IEEE Transaction on Medical Imaging, Vol. 18, No. 8, pp. 712-721, 1999.

7. P. Hellier, C. Barillot, E. Memin, P. Perez. Hierarchical Estimation of a Dense Deformation Field for 3-D Robust Registration. In: IEEE Transactions on Medical Imaging, Vol. 20, No. 5, pp. 388-402, 2001.

8. R. Shekhar, V. Zagrodsky. Mutual Information-based Rigid and Nonrigid Registration of Ultrasound Volumes. In: IEEE Transactions on Medical Imaging, Vol. 21, No. 1, pp. 922, 2002.

9. C.R. Meyer, J.L. Boes, B. Kim, P.H. Bland, G.L. Lecarpentier, J.B. Fowlkes, M.A. Roubidoux, P.L. Carson. Semiautomatic registration of volumetric ultrasound scans. In: Ultrasound in Medicine and Biology, Vol. 25, No. 3, pp. 339-347, 1999.

10. R.W. Prager, A. Gee, L. Berman. StradX: Real-Time Acquisition and Visualization of Freehand Three-Dimensional Ultrasound. In: Medical Image Analysis, Vol. 3, No. 2, pp. 129-140, 1999.

11. M.M.J. Letteboer, M.A. Viergever, W.J. Niessen. Rigid registration of 3D ultrasound data of brain tumours. In: Computer Assisted Radiology and Surgery, 2003. In Press.

12. www.itk.dk/image/nablavision

13. M.M.J. Letteboer, W.J. Niessen, P.W.A. Willems, E.B. Dam, M.A. Viergever. Interactive Multi-Scale Watershed Segmentation of Tumors in MR Brain Images. In: Proceeding of Interactive Medical Image Visualization and Analysis, Editors: S.D. Olabarriaga, W.J. Niessen, F.A. Gerritsen., pp. 11-16, 2001. 